\title{
CONF-941144--156
}

\section{FABRICATION OF OPTICAL CHANNEL WAVEGUIDES IN THE GaAs/AIGaAs SYSTEM BY MEV ION BEAM BOMBARDMENT}

T. Taylor", D. Ila*, R. L. Zimmerman", P. R. Ashley"* and D. B. Poker"*

"Center for Irradiation of Materials, Alabama A\&M University, Department of Physics

Normal, Alabama 35762

"Weapons Sciences Directorate

Research, Development and Engineering Center, U. S. Army Missile Command

Redstone Arsenal, Alabama 35898

-.*. Solid State Division, Oak Ridge National Laboratory

Oak Ridge, Tennessee 37831

\section{ABSTRACT}

We have fabricated optical channel waveguides in planar GaAs/AlGaAs waveguides using 10 $\mathrm{MeV}$ oxygen ions at a fluence of $3 \times 10^{13}$ and $3 \times 10^{14}$ ions $/ \mathrm{cm}^{2}$. Although disordering of GaAs/AlGaAs quantum well structures has previously been reported, to the best of the authors' knowledge the fabrication of channel waveguides using high energy oxygen bombardment has not been demonstrated in this material system. This technique may provide a totally new concept of localized material modifications in GaAs/AlGaAs waveguides by creating compositional disordered regions that act as optical confinement channels. The masking technique used to provide selective disordering of the planar waveguide structures will be presented. Optical measurements were performed on the channel waveguides at a wavelength of $1.3 \mu \mathrm{m}$.

\section{INTRODUCTION}

There is increasing interest in utilizing III-V waveguides in optoelectronic devices as either active or passive components. The interest has been motivated by the potential for monolithic integration of optoelectronic components. In a waveguide, a focused laser beam is confined in one (planar) or two (channel) dimensions to values of the order of the wavelength of light used for distances determined primarily by the propagation losses. These are typically millimeters to centimeters in integrated optical waveguides. Low loss electro-optical waveguides have potential use as interferometric modulators [1]. Channel waveguides in particular are attractive for compounded integration because they can be fabricated from a variety of materials and can readily be tailored to meet a specific task requirement by a number of methods. There have been numerous techniques developed for fabricating channel waveguide geometries in the III-V system for application into optoelectronic integrated circuits (OEICs) $[2,3]$.

Ion beam processing is an established technique used to create localized material modifications in the band gap and refractive index of GaAs/AlGaAs quantum well waveguides. Changes in the refractive index can provide lateral waveguide confinement of laser light through the guiding region. Ion beam processing offers the advantage of producing precisely defined alteration in specified regions around a selective mask while leaving other regions undisturbed. The majority of the literature in this area of research concentrates on using energy in the range 
of 5 to $800 \mathrm{keV}$ for the formation of disordered regions in the GaAs/AlGaAs system [4-6]. Low energy disordering schemes of this nature require a high temperature post-annealing procedure $\left(\sim 850^{\circ} \mathrm{C}\right.$ for $\left.\mathrm{GaAs} / \mathrm{AlGaAs}\right)$ [7] to enhance the compositional disordering due to the shallow penetration depth of the bombarded ions. The high temperature annealing step can restrict future device fabrication processing and the shallow deposited ions can promote undesirable doping effects within the layers. Efforts to minimize these factors prompted our investigation into the use of high energy ion beam bombardment to alter the optical properties of planar GaAs/AlGaAs waveguides. The compositional disordering that defines the channel edges in the layers is accomplished by energy transferred from the ions through electronic collisions. High energy ions penetrate into the layered structure with the end range occurring in the substrate layer. The final distribution of ions within the substrate can produce high resistivity regions for device isolation purposes [8], in addition to the disordering of the layers.

In this article, we provide a preliminary report on the direct method employed to fabricate channel waveguides in planar $\mathrm{GaAs} / \mathrm{AlGaAs}$ waveguide structures without subsequent thermal annealing. Optical measurements confirming the existence of channel regions created during the high ion energy bombardment process will be presented.

\section{EXPERIMENTAL}

The waveguide structure consisted of a high index guiding layer (core) bounded on the top and bottom by layers of $\mathrm{Al}_{x} \mathrm{Ga}_{1-x}$ As which formed the low index claddings, or vertical confining layers. The $\mathrm{Al}$ mole fraction for the $\mathrm{Al}_{\mathrm{x}} \mathrm{Ga}_{1-\mathrm{x}} \mathrm{As}$ cladding layers in the waveguide structure was $x=0.4$. The waveguide was grown by molecular beam epitaxy (MBE) on an exact (100) oriented Si-doped $\left(2 \times 10^{18} / \mathrm{cm}^{3}\right)$ GaAs substrate. The remaining planar GaAs/AlGaAs waveguide structure was grown as follows: A $0.5 \mu \mathrm{m}$ thick $\mathrm{n}^{+}$-type GaAs buffer layer with $\mathrm{Si}$ doping equivalent to the substrate was followed by $1.0 \mu \mathrm{m}$ of $\mathrm{n}$-type AlGaAs doped with $\mathrm{Si}$ to $5 \times 10^{17} / \mathrm{cm}^{3}$. This layer was followed by $0.5 \mu \mathrm{m}$ of n-type AlGaAs doped with Si to $1 \times 10^{17} / \mathrm{cm}^{3}$. The final layer of the lower cladding was $0.2 \mu \mathrm{m}$ of undoped AlGaAs. The guiding region consists of a single layer of undoped GaAs with a thickness of $0.3 \mu \mathrm{m}$. The upper cladding contained a $0.3 \mu \mathrm{m}$ thick layer of undoped $\mathrm{AlGaAs}$ followed by a $0.5 \mu \mathrm{m}$ layer of p-type $\left(\mathrm{Be}=1 \times 10^{17} / \mathrm{cm}^{3}\right)$ AlGaAs. The final layer was a $\mathrm{p}^{+}$-type $\left(\mathrm{Be}=1 \times 10^{18} / \mathrm{cm}^{3}\right) \mathrm{GaAs}$ cap with $0.1 \mu \mathrm{m}$ thickness (refer to figure 1). The doping profile of the waveguide was designed to provide minimal losses due to free-carrier absorption. The planar waveguides used in this study were shown to operate as suitable waveguides at a wavelength of $1.3 \mu \mathrm{m}$

Since the compositional disordering was desired in selective areas of the layers, there was a need to mask the surface of the samples to protect portions of the waveguide structure from ion penetration. Gold was chosen as the masking element because it has a large stopping power which results from its large atomic mass. The effective thickness required for the $\mathrm{Au}$ mask was determined from the incident ion's projected range values calculated with the TRIM [9] code. The masking method, produced at the MICOM Laboratory, was designed to create a series of electroplated Au stripes (5-8 $\mu \mathrm{m}$ in mask width) along the length of the samples. Instead of depositing the $\mathrm{Au}$ mask stripes directly onto the waveguide surface the following electroplating technique was applied: 1) a $0.02 \mu \mathrm{m}$ thick layer of $\mathrm{Cr}$ was evaporated on the waveguide surface, followed by a $0.25 \mu \mathrm{m}$ layer of $\mathrm{Au}$, followed by a final layer of $0.02 \mu \mathrm{m} \mathrm{Cr}$. The lower $\mathrm{Cr}$ layer acted as a diffusive barrier between the gold layer and the $0.1 \mu \mathrm{m}$ GaAs cap. 


\section{DISCLAIMER}

This report was prepared as an account of work sponsored by an agency of the United States Government. Neither the United States Government nor any agency thereof, nor any of their employees, makes any warranty, express or implied, or assumes any legal liability or responsibility for the accuracy, completeness, or usefulness of any information, apparatus, product, or process disclosed, or represents that its use would not infringe privately owned rights. Reference herein to any specific commercial product, process, or service by trade name, trademark, manufacturer, or otherwise does not necessarily constitute or imply its endorsement, recommendation, or favoring by the United States Government or any agency thereof. The views and opinions of authors expressed herein do not necessarily state or reflect those of the United States Government or any agency thereof. 


\section{DISCLAIMER}

Portions of this document may be illegible in electronic image products. Images are produced from the best available original document. 
The upper $\mathrm{Cr}$ layer was used to improve photoresist adhesion. 2) several microns of Shiply 1818 photoresist were spin coated onto the $\mathrm{Cr} / \mathrm{Au} / \mathrm{Cr}$ metal mask and baked; 3 ) a second layer of spin coating photoresist was applied until the total photoresist thickness measured $5.2 \mu \mathrm{m}$ and was then baked; 4) the photoresist was patterned by exposure to UV light and then developed; 5) the top layer of $\mathrm{Cr}$ was then etched away using chrome etchant CEN300 to expose the Au layer; 6) Au was electroplated in a solution of Au cyanide at a temperature of $70^{\circ} \mathrm{C}$ to a thickness of $4.2 \mu \mathrm{m}$ under lateral confinement by the photoresist; 7) the final step was to dissolve the photoresist away with acetone. After the masking process, the entire bulk waveguide was cut into smaller samples using a dicing saw.

The ion disordering was carried out at room temperature using $10 \mathrm{MeV} \mathrm{O}^{5+}$ ions at a fluence of $3 \times 10^{13} / \mathrm{cm}^{2}$ and $3 \times 10^{14} / \mathrm{cm}^{2}$. The ion beam current was held constant at $700 \mathrm{nA}$ for both samples. The ion beam experiments were performed at Oak Ridge National Laboratory, Oak Ridge, Tennessee, using a 1.7 MV General Ionex Tandem ion accelerator. The use of a tandem accelerator ensured a more stable beam because of the utilization of solid state charging systems. Once the ion beam experiments were completed the channel waveguides were characterized by performing optical transmission and propagation loss measurements.

\section{RESULTS \& DISCUSSION}

The two planar GaAs/AlGaAs waveguide samples used in this study had an area of about $0.32 \mathrm{~cm}^{2}$. The waveguide and mask profile are illustrated in Figure 1. Also shown, is the ion bombardment procedure used to fabricate the channel regions. The compositional disordering of the GaAs/AlGaAs layers was induced from the energy deposited by the ions as they penetrate through the structure. The incident energy of $10 \mathrm{MeV}$ for oxygen was chosen to yield the maximum electronic energy loss near the interface separating the top cladding layer (i-AlGaAs) and the GaAs guiding layer using stopping power calculations obtained from the TRIM code.

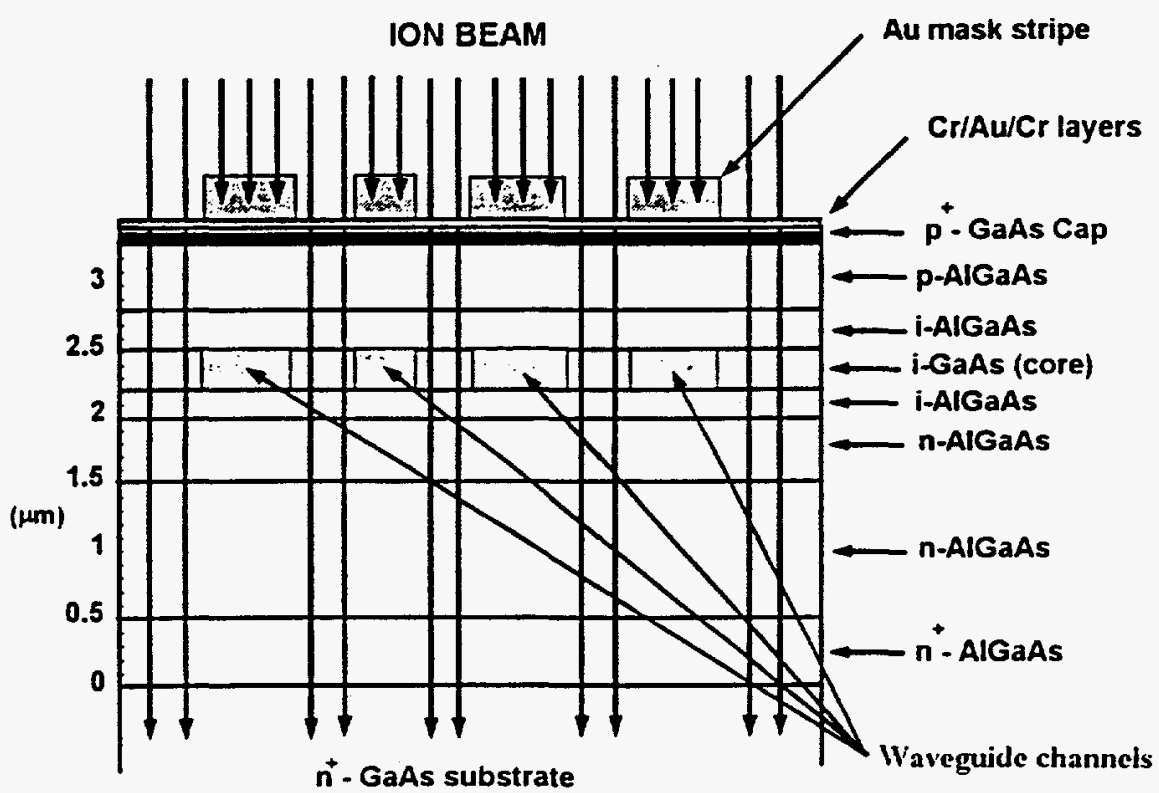

Figure 1. Schematic of the waveguide channels fabricated by high energy ion bombardment.

The exact mechanism by which the disordering process occurs is not fully understood at this time. Current attempts to formulate a quantitative model have been unsuccessful. However, 
there does appear to be a number of dynamic physical effects taking place. From the evidence acquired during optical measurements, disordering of the GaAs/AlGaAs layers has occurred in regions where ion penetration occurred. The regions directly adjacent to the channel regions were behaving as lateral optical confinement barriers. The result drawn from this observation is that the disordering has effectively decreased the refractive index of the bombarded layers. The extent to which the refractive index has been altered will be the focus of further analysis. The Au mask stripes were not removed prior to optical measurements because they will serve as high speed switching electrodes during future experiments.

An interesting feature expected during the high energy bombardment process has been observed from the light transmitted at $1.3 \mu \mathrm{m}$ through the GaAs substrate shown in Figure 2. The GaAs substrate, which is relatively transparent in the range of $\lambda_{o} \sim 0.87$ to $12 \mu \mathrm{m}$, shows that there was a dramatic absorption increase in certain sections within the top portion of the substrate.

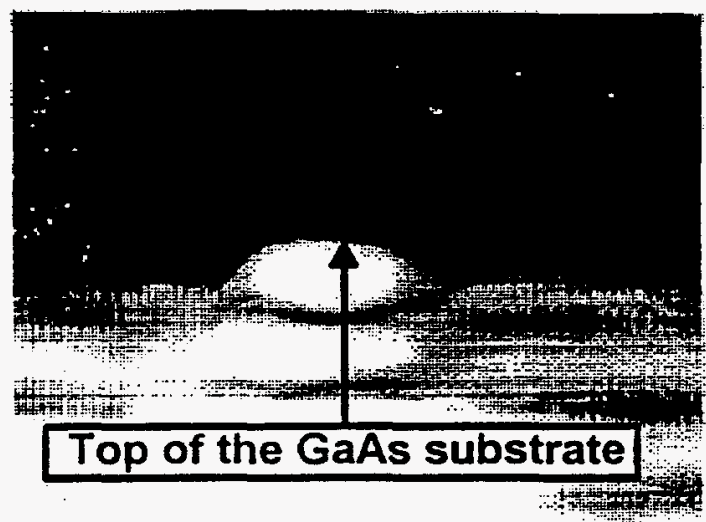

Figure 2. Transmission image of light at $1.3 \mu \mathrm{m}$ through the GaAs substrate.

These increased absorption regions appeared to coincide with the points where the ions penetrated through the layers. Using the TRIM code, we determined the projected range of the $10 \mathrm{MeV}$ oxygen ions through the waveguide structure to be about $5.1 \mu \mathrm{m}$. The estimated thickness of the substrate absorption region was about $1.9 \mu \mathrm{m}$; adding this value to the vertical thickness of the waveguide layers, a value of $5.6 \mu \mathrm{m}$ was obtained. This result showed that there was good agreement between the expected range predicted by the TRIM code and the experimentally observed ion range. An obvious advantage to using higher energies would be that the majority of damage produced by nuclear interactions occurring between the ions and the target atoms is concentrated within the GaAs substrate. This disordering technique could serve to minimize undesired doping effects and remove the restriction associated with the type of ion species used since the induced disordering process occurs prior to ion stoppage.

The fabrication of the channel regions was verified by using the optical setup described below. The channel waveguide mode profiles were measured using this setup. The optical properties of the channel waveguides were measured by end fire coupling a focused laser diode source at a wavelength $1.3 \mu \mathrm{m}$. A polarizing beam splitter was used to quickly change between TE and TM modes during analysis. The image of the optical output mode from the waveguide was captured using an image analysis system. A lens was used to image the output onto an IR camera which scanned the captured image yielding width and depth mode profiles. Single mode operation was observed for both TE and TM modes on channels with a width of 7 $\mu \mathrm{m}$. No planar guiding was observed in the adjacent ion bombarded regions. Figure 3 shows a transmission mode output along with the resulting three dimensional plot of the relative 
intensity mode profile for this waveguide. The mode size at the FWHM was $2.8 \times 7.3 \mu \mathrm{m}$. The propagation losses were determined by measuring the insertion loss and then subtracting the

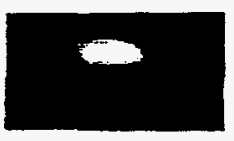

IMPS

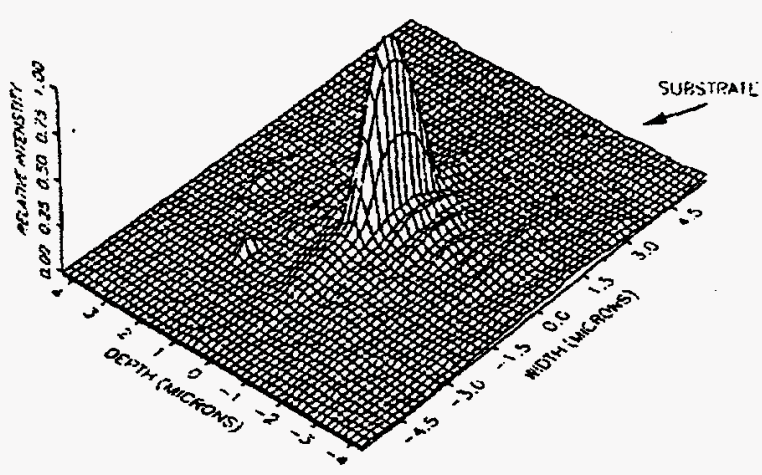

Figure 3. The top figure shows the transmission mode output from one of the waveguide channles. The lower figure shows a 3-D plot of the relative intensity mode profile (this data is typical of both the samples).

estimated coupling loss due to mode mismatch and Fresnel Reflection. The excess loss due to the channel waveguide was thus determined to be about $7 \mathrm{~dB} / \mathrm{mm}$ for the sample having $3 \times 10^{14}$ ions $/ \mathrm{cm}^{2}$. The other sample $\left(3 \times 10^{13}\right.$ ions $\left./ \mathrm{cm}^{2}\right)$ yielded higher losses of about $15 \mathrm{~dB} / \mathrm{mm}$. These relatively high losses may have resulted in part, from the weak waveguiding as evident by the mode size compared to the core dimensions. A thicker waveguide core would improve the vertical guide strength while a higher ion fluence could improve the lateral confinement by increasing the refractive index difference $\Delta \mathrm{n}$.

Our demonstration that optical channel waveguides have been produced by $\mathrm{MeV}$ ions passing through planar GaAs/AIGaAs waveguides can be explained by the formation of an optically modified material where the ions have passed, probably by the relocation of atoms across the layer interfaces along the ion tracks with limited lateral movement. Three mechanisms for this relocation can be proposed: Thermal diffusion during the transient temperature rise from energy transferred to the lattice, ballistic recoil of atoms after a large angle scattering of the incident ions with the atomic nuclei, and rapid displacement of ionized atoms in the Coulomb fields generated when electrons are removed by the passage of the incident ions.

Thermal diffusion certainly occurs if the temperature is high enough for a sufficient time. However, observation of channel edges can be explained only if atoms diffuse faster along the concentration gradient than heat diffuses laterally, a situation inconsistent with our knowledge of the diffusion constants. The dislocation of a sufficient number of atoms by nuclear recoil is also quantitatively inadequate to account for the change in the refractive index produced by the level of ion fluences used. Furthermore, the incident energy and ion species were chosen such that a large electronic energy loss could be obtained with a minimum of nuclear interactions. At the end of the ion range, these nuclear interactions dominate, and the disorder that they produce is useful to produce a higher resistivity layer in the substrate for future incorporation into optoelectronic devices.

The movement of heavy atoms in crystals is known to be the mechanism for color center 
production in crystals using $\mathrm{X}$-rays where nuclear recoil and heat production is absent but where charges are separated by the photoelectric effect. MeV ions similarly separate charge by a large transfer of electronic energy to atomic electrons. At the interface between the top (i-AlGaAs) cladding layer and GaAs core layer a significant change in electronic stopping power can cause a transport of electrons across that interface, leaving residual ionized atoms in a strong electric field. The resulting coulomb forces may be responsible for the transport of atoms across the AlGaAs/GaAs interface analogous to the sputtering of atoms at a free surface of an insulator being bombarded by ions.

\section{CONCLUSIONS}

We have fabricated optical channel waveguides in planar GaAs/AlGaAs waveguides using high energy $\mathrm{MeV}$ ion beam disordering. The reduction in the propagation loss associated with increased ion beam fluence suggests that enhanced disordering has occurred. This observation represents a significant finding, which will be explored with further effort. By optimizing the ion beam parameters required to induce disordering, this technique, with further development, could become a valuable method for fabricating channel waveguides in the $\mathrm{GaAs} / \mathrm{AlGaAs}$ system. The preliminary results from this study can be complemented by utilizing a well known analytical technique such as SIMS to investigate the extent to which the layers have been disordered.

\section{ACKNOWLEDGMENTS}

The authors would like to especially thank Eric Webster for accomplishing the masking procedure at the MICOM Laboratory and Dale Hensley for providing technical assistance during the ion beam experiments at ORNL. This project is supported in part by NSF-MERCE under grant number RII880291, by the Division of Materials Science, U.S. Department of Energy, under contract DE-AC05-84OR21400 with Martin Marietta Energy Systems, Inc., and the Howard J. Foster Center for the Irradiation of Materials at Alabama A\&M University.

\section{REFERENCES}

1. J.S. Cites and P.R. Ashley, IEEE J. Lightwave Technol. 12 (7), 1167 (1992).

2. W D. Laidig, N. Holonyak Jr., M.D. Camras, K. Hess, J.J. Coleman, P.D. Dapkus and J. Bardeen, Appl. Phys. Lett. 38, 776 (1981).

3. F. Julien, P.D. Swanson, M.A. Emanuel, D.G. Deppe, T.A. DeTemple, J.J. Coleman, and N. Holonyak Jr., Appl. Phys. Lett. 50 (14), 866 (1987).

4. W. Xia, S.C. Lin, S.A. Pappert, C.A. Hewett, M. Fernandes, T.T. Vu, P.K.L. Yu and S.S. Lau, Appl. Phys. Lett. 55, 2020 (1989).

5. W. Xia, S.N. Hsu, C.C. Han, S.A. Pappert, B. Zhu, C. Cozzolino, P.K.L. Yu, S.S. Lau, D.B. Poker, C.W. White, and S.A. Schwarz, Nucl. Instr. and Meth. B57/60, 491 (1991).

6. M. O'Neill, A.C. Bryce, J.H. Marsh, R.M. De La Rue, J.S. Roberts, C. Jeynes, Appl. Phys. Lett. 55 (14), 1373-1375 (1989).

7. T. Venkatesan, S.A Schwartz, D.M. Hwang, R. Bhat. M. Koza, H.W. Yoon, P. Mei, Y. Arakawa and A. Yariv, Appl. Phys. Lett. 40, 904 (1982).

8. S.J. Pearton, Nucl. Instr. and Meth. B59/60, 970-977 (1991).

9. J. F. Ziegler, J. P. Biersack and U. Littmark, The Stopping and Range of Ions in Solids (Pergamon Press Inc., New York, 1985). 